\title{
Riflessi della corrente neodialettale nella narrativa contemporanea pugliese. Analisi sociolinguistica
}

\section{The phenomenon of "neodialettalità" in contemporary prose of Apulia region. Sociolinguistic analysis}

\author{
Ingeborga Beszterda \\ Université Adam Mickiewicz, Poznań \\ e-mail: borging@amu.edu.pl
}

\begin{abstract}
The phenomenon of "neodialettalità" is examined from to perspectives. Initially the term was used to describe works of Italian poets in vernacular languages. Such choice of language was a result of Italian poets' protest in 1960s against gradual disappearance of vernacular languages and cultural traditions inherent to them. Currently the phenomenon of "neodialettalità" is a consequence of revolutionary sociocultural changes which took place over the last 30-40 years. Those changes did make Italian commonly used but at the cost of vernacular languages which for decades have been pushed to the margin and considered to be a symbol of backwardness or social degradation. It should come as no surprise then that those languages degenerated (or degraded, went through a crisis - author's comment) and as a result cultural values inherent to them did too. Currently we can observe in Italian society a desire to partly resurrect those traditions though popularization of vernacular languages in many aspects of social life: in literature, advertising, graffiti, newspapers, websites etc. In this paper the author analyses novels of two authors from Apulia region whose works, both in terms of language and sociopragmatics, refer to the phenomenon of "neodialettalità".
\end{abstract}

Keywords: "neodialettalità", Italian vernacular languages, Italian dialects, code-switching, code-mixing, Italian speech community

L'obiettivo che ci si prefigge di conseguire nel quadro del presente contributo è quello di dimostrare che la presenza dei tratti dialettali nella narrativa contemporanea potrebbe essere ascritta al fenomeno della cosiddetta neodialettalità. A sostegno 
della tesi sulla dialettalità riflessa nella narrativa delle due scrittrici pugliesi, Gabriella Genisi e Annalucia Lomunno, si divisa di presentare un'indagine abbastanza dettagliata, pur tuttavia non esaustiva, dell'uso del dialetto nei romanzi esaminati. L'analisi degli elementi dialettali ivi individuati si articola in due parti: una focalizzata su tratti linguistici di natura fonetica e morfosintattica, e l'altra dedicata alle funzioni discorsive dell'uso alternato dei due codici sotto forma di code-switching, code-mixing o prestito. L'indagine verrà integrata con le considerazioni relative alla situazione sociolinguistica d'Italia con particolare sguardo rivolto alle relazioni tra lingua nazionale e dialetti.

\section{NEODIALETTALITÀ, ORIGINI DEL FENOMENO}

Il termine neodialettalità fu inizialmente impiegato per designare una sorta di corrente letteraria accomunante poeti italiani che negli anni Sessanta avevano eletto il dialetto in alternativa ad una coeva ed egemonica poesia in lingua. Fu una reazione contro "la distruzione livellatrice di una lingua sempre più appiattita sui livelli medio-bassi di uno standard tecnologico" (Paccagnella, 1994 : 535) o addirittura come segno di "protesta contro la ruspa centralizzante che mira a distruggere ogni dialetto" (Mengaldo, 1994 : 235). È degno di nota osservare che il risveglio della poesia dialettale coincise con la decadenza e la progressiva scomparsa dei dialetti accompagnata dalla distruzione delle culture tradizionali sotto l'influsso del rapido progresso economico, radicali cambiamenti socioculturali e massiccia diffusione dell'italofonia su larga scala. In questo contesto, i dialetti nativi in quanto lingue di un mondo sull'orlo di estinzione sembrarono strumenti idonei all'espressione lirica "di un disagio esistenziale vissuto come dramma personale e collettivo" (De Blasi, 2010 : 363). Si noti in proposito, che nell'esame delle problematiche concernenti il fenomeno della neodialettalità in Italia non si può prescindere dallo sfondo socioculturale e sociolinguistico del paese che verrà delineato qui di seguito.

\section{SITUAZIONE SOCIOLINGUISTICA IN ITALIA}

Tra gli anni Sessanta e Settanta, nel contesto dei dati statistici riportati dall'ISTAT che dimostravano in maniera inequivocabile la progressiva perdita di vitalità dei dialetti alcuni studiosi (Berruto, $1994: 18$ ) avevano addirittura ipotizzato una loro imminente scomparsa o morte sotto la pressione di una progressiva diffusione della lingua nazionale, favorita dai mass media e dal sistema scolastico nonché dalla negativa percezione del dialetto presso la comunità italiana. In effetti, fino agli anni Ottanta il dialetto ha avuto la connotazione "di collocazione sociale bassa, di svantaggio culturale, di ridottissimo prestigio, di discriminazione sociale", 
osserva Grassi (2001 : 254) facendo notare al contempo, che tale atteggiamento sociale è stato sicuramente favorito dall'affermazione della lingua nazionale presso sempre più vasti strati della popolazione.

Tuttavia, in modo del tutto inopinato, i sondaggi DOXA pubblicati nel 1992 hanno palesato "un arresto parziale della chiara tendenza all'abbandono del dialetto rilevabile nei sondaggi precedenti, e un aumento del comportamento bilingue" (Berruto, 1994 : 22). Bisogna osservare inoltre che le stesse indagini hanno attestato un incremento dei comportamenti linguistici propensi ad incrociare nell'uso l'italiano e dialetto. Questa tendenza si è rivelata in aumento e trova la sua conferma non solo nelle ricerche di singoli studiosi (Marcato, 2001; Grassi, 2002; Marcato, 2002; Sobrero and Miglietta, 2006; D'Agostino, 2007) ma viene anche comprovata dalle inchieste ISTAT pubblicate negli ultimi anni. Si consulti la tabella che presenta i dati relativi alle preferenze dei parlati per determinati codici in diverse situazioni comunicative.

\begin{tabular}{|l|r|r|r|r|r|r|}
\hline \multirow{2}{*}{ Tipo di linguaggio usato } & \multicolumn{2}{|c|}{ In famiglia } & \multicolumn{2}{c|}{ Tra amici } & \multicolumn{2}{c|}{ Con estranei } \\
\cline { 2 - 7 } & 1988 & 2012 & 1988 & 2012 & 1988 & 2012 \\
\hline Solo o prevalentemente italiano & 41,5 & 53,1 & 44,6 & 56,4 & 64,1 & 84,8 \\
\hline Solo o prevalentemente dialetto & 32,0 & 9,0 & 26,6 & 9,0 & 13,9 & 1,8 \\
\hline Sia italiano che dialetto & 24,9 & 32,2 & 27,1 & 30,1 & 20,3 & 10,7 \\
\hline
\end{tabular}

Tra gli anni 1988-2012 si assiste all'aumento dell'uso prevalente dell'italiano in tutti i contesti, ma si nota un suo notevole incremento con gli estranei. Questo fenomeno è accompagnato, come si segnalava prima, da un considerevole calo dell'uso esclusivo del dialetto. Progrediscono invece gli usi alternati italiano dialetto nei contesti famigliari e con gli amici, il fatto già sostenuto dagli studiosi e comprovato dalle statistiche.

Nell'analisi dei dati bisogna tuttavia tenere conto di disparità e/o contrasti di natura sociolinguistica tra diverse regioni della Penisola. Vale la pena sottolineare comunque che l'uso alternato dei due sistemi linguistici viene privilegiato dalle comunità del Mezzogiorno d'Italia, in particolare in Sicilia e in Puglia. Dai rilevamenti del 2000 (Sobrero and Miglietta, 2006) risulta rispettivamente il 42,5\% e il 49,8 in famiglia, nonché il 44,2\% e 48,6 con gli amici. Berruto (2005 : 216) osserva in proposito che la selezione del codice da parte del parlante non avviene in maniera del tutto spontanea, bensì è sottoposta a determinate restrizioni in quanto dipende dal dominio, dall'interlocutore, dall'argomento o dal contesto comunicativo. D'altra parte, Grassi (2001 : 254) constata il dilagare del fenomeno presso diversi strati sociali come una risorsa comunicativa ed espressiva. Secondo Sobrero (1998: 19) una relativa facilità con la quale avviene il passaggio dalla lingua al dialetto permet- 
te ai parlanti di usarli come se fossero i registri diversi della stessa lingua. Va ribadito tuttavia che tali comportamenti verbali sono diventati possibili e frequenti grazie al cambiamento degli atteggiamenti dei parlanti nei confronti del dialetto.

A titolo riassuntivo possiamo affermare seguendo Sobrero and Miglietta (2006 : 155) che a partire dagli anni Novanta del secolo scorso, il dialetto ha riacquistato dignità riguadagnando una collocazione stabile all'interno del repertorio linguistico della comunità italiana. Nell'opinione generale, questa inversione di tendenza è stata determinata dalla diffusione esponenziale della lingua nazionale iniziata negli anni Cinquanta e portata a compimento proprio negli anni Ottanta-Novanta. Il raggiungimento di una sufficiente competenza in lingua da parte della maggior parte dei parlanti ha riscattato il dialetto dallo stigma secolare. Berruto (2006) sottolinea che il dialetto oggi

non risulta più $[\ldots]$ codice tipico dei ceti bassi, simbolo di ignoranza e di esclusione dal mondo moderno, legato allo svantaggio sociale e culturale, portatore di connotazioni socio-culturalmente negative, ma si configura come una tastiera di arricchimento espressivo, accanto all'italiano, per tutti i parlanti bilingui. Non è più stigmatizzato socialmente come varietà unicamente bassa, nella consapevolezza e nelle rappresentazioni della comunità parlante: sapere un dialetto è un valore positivo, una possibilità in più nel repertorio comunicativo individuale, da sfruttare per i suoi valori particolari [...] (p. 121).

Tenendo conto di quanto presentato sopra non deve stupire quindi l'ipotesi avanzata da Berruto (2002 : 48): "un motto dell'Italia alle soglie del terzo Millennio sembra essere "ora che sappiamo parlare italiano, possiamo anche (ri)parlare dialetto"”. Antonelli (2006 : 97) conclude ammiccando "Il dialetto non è più un delitto".

\section{NEODIALETTALITÀ E LE SUE MANIFESTAZIONI OGGI}

Il quadro sociolinguistico tracciato qui sopra permette di elucidare le condizioni dell'avvento di una nuova ondata di neodialettalità. Il fenomeno in questione è stato favorito da una fondamentale svolta negli atteggiamenti degli italiani nei confronti del dialetto determinata dall'affermazione generalizzata dell'istruzione scolastica e della lingua nazionale. Ne consegue che il dialetto, tacciato, tra l'altro, di rappresentare un grave ostacolo all'acquisizione della lingua nazionale, si è riscattato dal decennale stigma sociale che lo colpiva. Berruto (2006) osserva in proposito:

il dialetto non risulta più, non è più vissuto come codice tipico dei ceti bassi, simbolo di ignoranza e di esclusione dal mondo moderno, legato allo svantaggio sociale, portatore di connotazioni socio-culturalmente negative, ostacolo alla promozione sociale (p. 120).

Si noti d'altra parte, che il mutamento degli atteggiamenti linguistici ha trasformato, a sua volta, i comportamenti verbali della comunità italiana. Proprio a partire dagli anni Ottanta-Novanta si assiste al rallentamento del processo di abbandono del 
dialetto, che diventa addirittura un'ulteriore risorsa discorsiva accanto alle strategie dell'alternanza o frammistione dei due codici. Berruto (2006) indica quattro funzioni attribuibili all'uso del dialetto: valore comunicativo effettivo, risorsa espressiva con intenti sostanzialmente ludici, rappresentazione simbolica ed ideologica dei mondi di riferimento e di valori socioculturali, raccolta di tradizioni a scopo folcloristico e museografico. In questo contesto sembra del tutto naturale che il fenomeno della nuova dialettalità si propaga in diversi strati sociali e in diversi ambiti della vita sociale, come p.es. fumetti, pubblicità delle tv locali e nazionali, denominazione di ristoranti, rubriche di messaggi dei settimanali a diffusione locale, cinema e letteratura, comunicazione mediata dal computer: mail, chat, sms e instant messaging (Grimaldi, 2006 : 429). A questo lungo elenco conviene aggiungere ancora il ricorso al dialetto (o elementi del dialetto) da parte del mondo della subcultura giovanile. Vanno menzionate in questo luogo principalmente le bande musicali che compongono le canzoni rap/reggae, iscrivendosi al filone simbolico-ideologico, come p.es. Almamegretta, Sud Sound System, Pitura Fresca, Mau Mau, Sa Razza Posse. Al polo opposto dei valori si situano i rappresentanti del filone lirico-espressivo (e anche ludico), ossia i cantautori citati da Coveri (2012) : De André, Daniele, De Sio, Jannacci, Battiato, Consoli.

La corrente neodialettale trova la sua rappresentazione anche nella narrativa contemporanea, avendo conquistato uno spazio privilegiato soprattutto nel genere poliziesco. Grazie alla fortuna di Andrea Camilleri, che negli anni Novanta aveva forse agito da battistrada agli usi linguistici locali sotto forma di dialetti e/o di italiani regionali, si assiste oggi ad una vera e propria proliferazione di romanzi gialli marcati in senso regionale, sia dal punto di vista linguistico che quello dell'ambientazione della trama. Si consulti la relativa analisi di Vignuzzi and Bertini Malgarini (2010) che non solo citano almeno una quindicina di autori di romanzi polizieschi ripartiti a seconda delle regioni, ma presentano anche un' analisi approfondita delle peculiarità del "giallo all'italiana". Un altro elenco di autori appartenenti al filone neodialettale (circa una ventina), limitati esclusivamente alla regione di Puglia, è stato stilato da Maria Carosella (2012). Naturalmente vi sono incluse le due scrittrici, i cui romanzi costituiscono l'oggetto della presente indagine.

\section{NEODIALETTALITÀ NELLA NARRATIVA PUGLIESE}

Nel quadro del presente contributo verranno presi in considerazione i romanzi di due scrittrici di origine pugliese: Gabriella Genisi e Annalucia Lomunno.

Gabriella Genisi è nata nel 1965 e abita a pochi chilometri da Bari. Ha creato il personaggio del commissario Lolita Lobosco, protagonista di cinque romanzi: La circonferenza delle arance (2010), Giallo ciliegia (2011), Uva noir (2012), Gioco pericoloso (2014) e Spaghetti all'Assassina (2015). 
Annalucia Lomunno è nata nel 1972 e attualmente vive in Spagna. È autrice di quattro romanzi Rosa sospirosa (2001), Nero Sud (2003), Troppe donne per un delitto (2009), Crimini del cuore (2016). Per lo scrupolo di coerenza dell'esposizione sono stati presi in considerazione soltanto due primi romanzi della Lomunno in quanto sono gli unici a soddisfare i requisiti prestabiliti nel presente contributo, cioè annoverabili al filone neodialettale.

L'idea di paragonare lo stile e le scelte linguistiche compiute proprio da quelle scrittrici non è stata casuale. Sono stati ponderati alcuni tratti che accomunano sia le autrici che i loro romanzi. In primo luogo, GG e AL hanno quasi la stessa età (una nata nel 1965 e l'altra nel 1972) nonché le stesse origini pugliesi. In secondo luogo, i romanzi, a nostro avviso, si iscrivono al filone neodialettale in quanto le storie ivi presentate sono ambientate negli strati medio-bassi della scala sociale il che costituisce un valido presupposto per un'indagine sociolinguistica basata su criteri possibilmente omogenei. Si tratta infatti di rilevare comportamenti linguistici assunti dai protagonisti in determinate situazioni comunicative che possono implicare o coadiuvare l'insorgenza di fenomeni del code-switching e/o del code-mixing. In quest'occasione si possono notare stratagemmi particolari, adoperati dalle rispettive scrittrici, per integrare le conversazioni con elementi dialettali o regionali. Per la chiarezza dell'esposizione ci si propone in primo luogo di sottoporre all'esame un sommario prospetto di tratti caratteristici del parlato pugliese elaborato dalle rispettive scrittrici.

\section{ASPETTI LINGUISTICI DEI ROMANZI DI GABRIELLA GENISI}

L'analisi che segue si concentra soprattutto su peculiarità fonetiche e morfosintattiche riscontrabili sia nella narrazione nelle conversazioni dei protagonisti. Per mancanza di spazio si tralascia l'analisi di elementi lessicali di origine dialettale o regionale. Per quanto riguarda aspetti fonetici vale la pena notare una notevole frequenza dei casi seguenti :

- raddoppiamento espressivo della consonante iniziale di parola

(1) Eddai, vieni qua attoniotuo e siediti un poco vicin'a mè. [CA : 84]

- pronuncia sonora delle occlusive sorde dopo nasale [angora], nel caso specifico accompagnata dall'apocope della vocale finale

(2) E aspétt' nu poc' angor', peppiacére, Lolì.[CA : 101

- troncamento dei nomi personali usati nel registro familiare come appellativi

(3) Devo chiederti una cosa, Dani, ti chiamo appunto per questo. [GC : 58]

- aferesi dell'articolo indeterminativo e dell'aggettivo dimostrativo "questo, questa"

(4) Un gesto gentile voleva essere, e questa si offende così... ecchhera 'na coltellata? [GC : 102]

(5) E che c'entra mo' 'sta cosa del letto, Lolì? [UN : 98] 
Quanto ai tratti morfosintattici che ricorrono con una certa regolarità conviene segnalare dei casi seguenti:

- impiego del cosiddetto "oggetto preposizionale"

(6) Sì, come se l'aereo aspetta a me, borbotta tutta sconsolata. [CA : 70]

- collocazione dell'aggettivo possessivo dopo il nome cui si riferisce

(7) Di' Antò, che c'è. E com'è che ti trovi nella stanza mia? E a fare che, poi?» [UN : 128]

- raddoppiamento espressivo dell'aggettivo

(8) Lolita nostra fa il commissario, e le entrano due o tremila euro al mese dritti dritti. [UN : 53]

- uso del Voi come pronome di cortesia

(9) Vit' commissà, che mammà non sa niente di questo fatto che vi sto per dire. E perciò voi dovete mantenere il segreto. [GC : 51]

- collocazione del predicato verbale nella posizione finale della frase

(10) Io però mi sono presa la mattina libera e fretta non ne tengo. [UN : 112]

- uso del che enfatico nelle interrogative

(11) Che te la porto 'na camomilla prima di andare a casa, Lolì? [CA : 106]

\section{ASPETTI LINGUISTICI DEI ROMANZI DI ANNALUCIA LOMUNNO}

In conformità all'ordine di presentazione adoperato sopra, anche in questa sezione, verranno prima indicati tratti fonetici più ricorrenti nei dialoghi cui seguirà l'esame di elementi morfosintattici.

- pronuncia affricata della sibilante dopo $\mathrm{n}, \mathrm{r}, \mathrm{l}$

(12) Le Baresi anonime sembrano più atte all'atto supremo. Assecondano felici il destino. Hanno addobbi e trasparenze Postal Market. La più carina parla male. Dice cose tipo: L'anno scorzo faccio la corza bevo un sorzo mi sento il piede perzo in una morza. [NS : 193]

- pronuncia sonora delle occlusive sorde in posizione postnasale, p.es. contenta [kon'denta]

(13) Complimenti, bravo, davero bravo. Sono davero condenta che ti anno dato cuesto premio. [NS : 136]

- troncamento dei nomi personali usati, nel registro familiare, come appellativi

(14) Accomodati, Save', su queste sorte di poltrone. [NS : 55]

- aferesi dell'aggettivo dimostrativo

(15) 'Sto telefonino è proprio aggarbato. [NS : 38]

- caduta della vocale finale atona (imitazione del dialetto di Taranto)

(16) Seh... stai bene tu... Filìp te lo raccomand! Che poi è andat in città, è diventat dentist e s'è fatt i miliard. E beh? Ti pare giust che io se mi voglio far la dentier da lui, mi devo vender la cas? [RS : 52] 
Quanto alle caratteristiche morfosinattiche tra i più rilevanti si osservino i casi seguenti:

- $\quad$ accusativo preposizionale

(17) Ueh, dotto'! Ma che ti prende? Perché l'hai trattata cosi male alla ragazza? [NS : 197]

- $\quad$ particella $c i$ con funzione attualizzante davanti al verbo avere

(18) Ci hai ragione. Si vede che sei una vera femmina intelligente. [NS : 91]

- $\quad$ raddoppiamento espressivo dell'aggettivo

(19) Venter e Voracissimus pronti alle scintille di riserva. Capelli pettinatipettinati. [RS : 84]

- $\quad$ impiego del che enfatico nelle interrogative

(20) Che sei scema? E ci frechiamo un sabato sera così? [RS : 122]

Come risulta da questa breve rassegna si possono osservare sostanziali similitudini rilevabili nella narrativa delle rispettive scrittrici quanto al trattamento di peculiarità del parlato pugliese il che dimostra la loro sensibilità linguistica degna di nota.

\section{LINGUA E DIALETTO IN CONTATTO}

In conformità ai dati statistici riportati sopra, nonostante la massiccia diffusione dell'italofonia e la regressione dell'uso esclusivo del dialetto, nelle regioni meridionali continua ad consolidarsi la tendenza ad alternare lingua e dialetto in contesti situazionali di media e bassa formalità (De Mauro, 2014 : 119). Questa tendenza trova la sua conferma e riflesso nella narrativa delle due scrittrici, pur con esiti diversi. Gabriella Genisi predilige il fenomeno del code-mixing, ricorrendo solo di rado al code-switching, mentre Annalucia Lomunno sembra propendere piuttosto per i prestiti dialettali. Per agevolare la lettura ai non pugliesi, la scrittrice aveva aggiunto in appendice dei due romanzi (RS, NS) rudimentali glossarietti.

Ambedue le autrici, a prescindere da peculiarità stilistiche adoperate, dimostrano tuttavia una notevole sensibilità per l'espressione linguistica di emozioni vissute e sperimentate dai protagonisti cercando soluzioni più appropriate per rendere verosimili, e allo stesso tempo, naturali i loro comportamenti verbali. Per mancanza di spazio non è possibile rendere conto di tutti i casi interessanti, ma si cercherà tuttavia di riportare alcuni esempi degni di nota. Esaminiamo prima alcuni frammenti di conversazioni (tra la commissaria Lolita Lobosco ed i suoi collaboratori o amici) in cui si possono rilevare determinate funzioni discorsive veicolate dall'alternanza tra lingua e dialetto, definite con il termine code-swiching. Si tratta rispettivamente di (1) esclamazione, (2) inizio e fine scherzoso della conversazione, (3) citazione.

(1) Mé, vattìn' Lolì! Che co' tté lo sappiamo non s'è mai potuto parlare di cos' assenziali... Buonanott'. [CA : 158] 
(2) - Sent'a mè, Lolì. Vediamo un poco, dimmi tu tieni niente in mano? insiste il questore. - Dottò, e che cosa posso tenere in mano [...] seguo una pista mia, diciamo. - Ahhh, mo' ho capito. Segui una pista tua. E dunque tu lo vorresti far scarcerare diciamo così, per simpatia? - Mannò, dottore, cosa va a penzare? [CA : 95]

(3) - A casa mia invece si usava il metodo antico. - Cioè? - Cioè mazz'e pannell fann'ì figli bèll. [GC : 24]

Bisogna sottolineare che nei romanzi di Gabriella Genisi le conversazioni in cui affiorano i fenomeni del code-mixing sono decisamente più frequenti rispetto a quelle del code-switching. Siccome il CM non è condizionato da mutamenti interni alla situazione discorsiva né svolge funzioni comunicative specifiche, l'autore gode di una fondamentale libertà ricorrendo ad un uso incrociato dei due codici. Per chiarezza di esposizione si riportano a titolo esemplificativo alcuni campioni di battute annoverabili al CM (a prescindere naturalmente dalle convenzioni di resa grafica dei passi dialettali adottate dall'autrice).

(4) E secondotè come ci vengono le idee annoi povere criste? [...] Siamo state costrette a fare di necessità virtù, chetticredevi che ci stavamo a divertire, noi sventurate? $[U V: 53]$

(5) Sto già a pezzi, quando sento suonare. Ecchipuoessere, già da quest'ora? Sono appena le sei... Ettipareva, chi? Antonio, mannaggiallui. [UN : 138]

La seconda scrittrice, Annalucia Lomunno, non era propensa a cimentarsi con l'alternanza dei codici, a quanto pare, perciò si era proposta un'altra soluzione, quella di inserire elementi dialettali nelle conversazioni sotto forma di prestiti. Per rendere l'idea delle scelte individuali della scrittrice nel trattamento del materiale linguistico si riportano qui sotto due frammenti dei dialoghi tratti dai rispettivi romanzi, RS e NS.

(6) Svegliati Rosa! Impara da me. Io so vivere. So gestire gli uomini. [...] I rattusi li schifo, mi concentro sui migliori e li tengo in pugno. Li uso, li faccio soffrire. Non mi faccio trattare a pezza!!! [RS : 17]

(7) Mi pare che una volta ho trovato una moneta, ma l'ho sbattuta a qualche parte che non mi ricordo più. A che sarmenghia serve? Che se m'ingappa la finanza devo azzoppare pure il resto. [...] Scià scià balliamo signorinelle! [NS : 100]

Come si vede, nonostante il ricorso delle rispettive scrittrici a strategie diverse, l'effetto finale è sostanzialmente simile in quanto permette di rendere conto dell'uso alternato dei due codici nelle conversazioni informali. Conviene sottolineare appunto che nei romanzi di Gabriella Genisi o Annalucia Lomunno l'impiego del dialetto riveste caratteri di stilizzazione del parlato, perlopiù dei parlanti appartenenti agli strati medio-bassi o inferiori, a volte legati al mondo della malavita. Oltre agli aspetti realistici, menzionati sopra, si notano tuttavia anche usi dialettali scherzosi, ludici ed espressivi o semplicemente per rendere il colore locale. A tal proposito 
conviene citare l'opinione di Maria Carosella (2012) riguardo allo stile letterario di Gabriella Genisi che, all'avviso di chi scrive, potrebbe riferirsi anche a quello sperimentato da Annalucia Lomunno:

In tutti questi casi $[\ldots]$ si tratta sempre di una dialettalità plausibile, non forzata, simile a quella di cui si servono normalmente i baresi di cultura medio-alta per interagire in modo amichevole, per fare battute, per esprimere sentimenti di varia (ed opposta) natura, $o$ anche solo nei pensieri (p. 72).

\section{CONSIEDRAZIONI FINALI}

A titolo riassuntivo conviene puntualizzare che la neodialettalità nella narrativa rappresenta, stando al distinguo introdotto da Bertini Malgarini and Vignuzzi (2002), il caso "dell'uso del dialetto in letteratura" e non "la letteratura dialettale". Questo assunto ricorda la discriminazione proposta da B.Croce (cited in Paccagnella 1994) tra l'uso dialettale nativo da parte di chi possiede il dialetto come l'unico strumento di comunicazione e l'uso dialettale riflesso da parte di chi predilige il dialetto (eventualmente stilizzato e/o codificato) pur avendo a disposizione uno strumento di maggior prestigio. In tal senso, la narrativa neodialettale contemporanea potrebbe sostanzialmente coincidere con il concetto crociano di "letteratura dialettale riflessa". Infatti, sembra plausibile l'opinione espressa da Bertini Malgarini and Vignuzzi (2010) a tal proposito:

la presenza del dialetto è sostanzialmente circoscritta a "macchie di colore" quasi spezia che esalti o almeno ravvivi il sapore di una prosa spesso stinta, quando non usuratamente banale. È un impiego riconducibile alla stilizzazione bozzettistica, specificatamente collegato alla definizione di situazioni o personaggi a connotazione "popolare" che si realizza, come è ovvio, soprattutto nelle battute dei dialoghi, oppure (e insieme) quando si voglia indulgere all'effetto "giocoso" o addirittura al comico (p. 234). 


\section{BIBLIOGRAFIA}

Antonelli, G. (2006). Lingua ipermedia. La parola di scrittore oggi in Italia. Lecce: Manni.

Berruto, G. (1994). "Come si parlerà domani: italiano e dialetto", in T. De Mauro (Ed.), Come parlano gli Italiani (pp. 15-24). Firenze: La Nuova Italia.

- (2005). "Che cosa ci insegna 'il parlare in due lingue'? Commutazione di codice e teoria linguistica e sociolinguistica" Rivista di Linguistica, XVII, 1, 2-14.

- (2006). "Quale dialetto per l'Italia del Duemila. Aspetti dell'italianizzazione e 'risorgenze dialettali' in Piemonte e altrove", in A. A. Sobrero, A. Miglietta (Eds.), Lingua e dialetto nell'Italia del Duemila (pp. 101-128). Galatina: Congedo.

Bertini Malgarini, P., Vignuzzi, U. (2002). Dialetto e letteratura, in M. Cortelazzo, C. Marcato, N. De Blasi, G. P. Clivio (Eds.). Dialetti italiani. Storia, struttura, uso (pp. 5-37). Torino: UTET.

- (2010). "La dialettalità nel 'giallo all'italiana': naturalismo o espressionismo?", in G. Ruffino, M. D’Agostino (Eds.), Storia della lingua italiana e dialettologia (pp. 233-248). Palermo: Centro di Studi Filologici e Linguistici Siciliani.

Carosella, M. (2012). "Cugini baresi di Montalbano", Esperienze letterarie, XXXVII, 2, 67-78.

De Blasi, N. (2010). "Uso letterario del dialetto", in Enciclopedia dell'italiano (pp. 363-365). Roma: Treccani.

- (2014). "Dialetto e varietà locali nella narrativa tra scelte tra scelte d'autore e storia linguistica di fine Novecento", in A. Dettori (Ed.), Dalla Sardegna all'Europa. Lingue e letterature regionali (pp. 15-38). Milano: Franco Angeli.

De Mauro, T. (2014). Storia linguistica dell'Italia repubblicana dal 1946 ai nostri giorni. RomaBari: Laterza.

Genisi, G. (2010). La circonferenza delle arance. Venezia: Sonzogno.

- (2011). Giallo ciliegia. Venezia: Sonzogno.

- (2012). Uva noir. Venezia: Sonzogno.

Grassi, C., Sobrero, A. A., Telmon, T. (2001). Fondamenti di dialettologia italiana. Roma - Bari: Laterza.

Grimaldi, M. (2006). "Parole antiche in suoni moderni: l'uso del dialetto salentino o nella musica giovanile hip-hop", in G. Marcato (Ed.), Giovani, lingue, dialetti (pp. 425-439). Padova: Unipress.

Lomunno, A. L. (2001). Rosa sospirosa. Casale Monferrato: Piemme.

- (2003). Nero Sud. Casale Monferrato: Piemme.

Lollini, M. (1992). "Aspetti della poesia neo-dialettale in Pasolini, Zanzotto e Loi”. NeMLA Italian Studies, 16, 67-84.

Mengaldo, P.V. (1994). Il Novecento. Bologna: Il Mulino.

Paccagnella, I. (1994). "Uso letterario dei dialetti”, in L. Serianni, P. Trifone (Ed.), Storia della lingua italiana. Vol. III: Le altre lingue (pp. 495-539). Torino: Einaudi.

Sobrero, A. A., Miglietta, A. (2008). Introduzione alla linguistica italiana. Roma - Bari: Laterza.

Sottile, R. (2013). Il dialetto nella canzone italiana degli ultimi venti anni. Roma: Aracne. 
